\title{
A Simple Method for Detection of Multiple Chemical-Specific IgGs in Serum Based on Dot Blotting
}

\author{
Mayumi Tsuji ${ }^{*}$, Hsu-Sheng Yu1,2, Yasuhiro Ishihara3 ${ }^{3}$, Toyohi Isse1, Nami Ikeda-Ishihara4, \\ Takuto Tuchiya ${ }^{1}$, Toshihiro Kawamoto ${ }^{1}$ \\ ${ }^{1}$ Department of Environment Health, University of Occupational and Environmental Health, Kitakyusyu, Japan \\ ${ }^{2}$ Department of Food Science, College of Agriculture, National Pingtung University of Science and Technology, Taiwan \\ ${ }^{3}$ Laboratory of Molecular Brain Science, Graduate School of Integrated Arts and Sciences, Hiroshima University, Higashi-Hiroshima, Japan \\ ${ }^{4}$ Division of Gene Research, Natural Science Center for Basic Research and Development, Hiroshima University, Higashi-Hiroshima, \\ Japan \\ Email: ^tsuji@med.uoeh-u.ac.jp
}

How to cite this paper: Tsuji, M., Yu, H.-S., Ishihara, Y., Isse, T., Ikeda-Ishihara, N., Tuchiya, T. and Kawamoto, T. (2016) A Simple Method for Detection of Multiple Chemical-Specific IgGs in Serum Based on Dot Blotting. Health, 8, 1645-1653. http://dx.doi.org/10.4236/health.2016.815160

Received: July 29, 2016

Accepted: December 3, 2016

Published: December 6, 2016

Copyright $\odot 2016$ by authors and Scientific Research Publishing Inc. This work is licensed under the Creative Commons Attribution International License (CC BY 4.0).

http://creativecommons.org/licenses/by/4.0/ c) (i) Open Access

\begin{abstract}
Plastic resins are known to cause occupational allergies. Therefore, serum-specific antibodies against plastic resins have been widely investigated as diagnostic markers for occupational allergies. In this study, we aimed to establish a convenient method for detection of multiple chemical-specific IgG antibodies in human serum based on dot blot analysis. Toluene diisocyanate (TDI), phthalic anhydride (PA), and formaldehyde (FA), which are frequently used to synthesize various resins, reacted well with lysine residues of human serum albumin (HSA) under alkaline conditions. Native polyacrylamide gel electrophoresis (PAGE) showed that the structures of chemical adducts of HSA were different from those of native HSA. Therefore, we performed dot blot assays using these adducts as artificial antigens. Serum samples from workers at plants utilizing plastic resins strongly reacted with TDI, PA, and FA adducts in HSA, while reduced signals were detecting using the serum from unexposed workers. These results suggested that dot blot assays using chemical-HSA adducts as antigens could be beneficial for simultaneously measuring multiple chemical-specific IgGs.
\end{abstract}

\section{Keywords}

IgG, Dot Blot Assay, Plastic Resin

\section{Introduction}

The production and consumption of plastics have increased worldwide. In 2010, plastic 
production reached 265 million tonnes worldwide, and approximately $5 \%$ of world plastic production is carried out in Japan [1]. Plastics are present ubiquitously throughout society and the environment. Most chemicals used for producing plastic polymers are derived from various types of nonrenewable crude oils, and some of these polymers are hazardous. The polymers ranked as most hazardous include polyurethanes (PURs), polyacrylonitriles (PANs), polyvinyl chloride (PVC), epoxy resins, and styrenic copolymers. Moreover, several additional types of polymers are considered hazardous, including phenol formaldehyde resins (PFs), unsaturated polyesters (UPs), polycarbonate (PC), polymethyl methacrylate (PMMA), and urea-formaldehyde resins (UFs) [2]. Toluene diisocyanate (TDI), which causes serious upper respiratory health problems [3], is the monomer used for PUR production, whereas phthalic anhydride (PA) is used for UP production, and formaldehyde (FA) is used for PF and UF production. FA can cause skin sensitivity, and PA can cause respiratory and skin sensitivity [2] [4]. Therefore, TDI, PA, and FA can induce occupational allergies in workers who come in contact with these chemicals [3] [5].

Chemical-specific IgE and IgG antibodies in serum, particularly those targeting TDI, have been widely investigated as diagnostic markers of occupational allergies. Recently, enzyme immunoassay methods, e.g., enzyme-linked immunosorbent assays (ELISAs), have been used worldwide for detection of these IgE and IgG proteins. Using ELISA, specific IgE has been shown to identify inhalation challenge-positive workers with $14 \%$ - $31 \%$ sensitivity and $89 \%$ - $97 \%$ specificity. The corresponding sensitivity and specificity estimates for specific IgG were reported to be $46 \%-72 \%$ and $74 \%$ - 92\%, respectively [6]. Based on measurements of sensitivity, specific IgG can be a useful diagnostic marker of occupational allergy. However, although several chemical-specific IgEs can be measured commercially, chemical-specific IgG cannot be measured commercially in Japan.

In general, plants handling plastic resins do not only use one type of chemical substance. Furthermore, workers may be exposed different combinations of chemical substances, particularly in small plants. Therefore, appropriate methods for measurement of chemical exposure should simultaneously utilize many inexpensive antibodies and have the capacity for analysis of small serum samples.

Accordingly, in this report, we established a method for simultaneous detection of multiple chemical-specific IgG antibodies in human serum with high sensitivity using dot blot assays. We used three major chemical substances used for the production of plastic resins, i.e., the isocyanate TDI and the carbonyl compounds PA and FA. Our findings provide important advancements in the detection of chemicals in serum samples.

\section{Materials and Methods}

\subsection{Measurement of the Amino Groups of HSA Conjugates}

The reaction of 2,4,6-trinitrobenzenesulphonic acid (TNBS) with primary amino groups within proteins was performed as previously described [7]. HSA conjugate (20 
$\mu$ g protein) was mixed with $100 \mu \mathrm{L}$ of $4 \% \mathrm{NaHCO}_{3}$, followed by the addition of $100 \mu \mathrm{L}$ of $0.01 \%$ TNBS. The reaction was carried out at $42^{\circ} \mathrm{C}$ for $2 \mathrm{~h}$ and then was stopped by the addition of $50 \mu \mathrm{L} \mathrm{HCl}$. The absorbance at $340 \mathrm{~nm}$ was measured. Known concentrations of L-lysine were used as standards.

\subsection{Native Polyacrylamide Gel Electrophoresis (PAGE)}

Qualitative assessment of structural changes in HSA conjugates was achieved by native PAGE performed on $8 \%(\mathrm{w} / \mathrm{v})$ acrylamide resolving gels. Ten micrograms of HSA or HSA conjugate was applied to the gel, and electrophoresis was performed. Protein bands were visualized by staining with Coomassie brilliant blue.

\subsection{Serum Samples}

Sera from workers handling plastic resin (e.g., TDI, FA, and PA) were collected in Kyusyu, Japan from 2013 to 2015. Sera from unexposed workers who live in Kyusyu were collected in 2014. After collection of blood samples, the blood was allowed to clot for approximately $3 \mathrm{~h}$ and then centrifuged at $2568 \times g 30 \mathrm{~min}$ to separate the serum portion of the blood. The sera were transferred to clean tubes and stored at $-80^{\circ} \mathrm{C}$ until use. The study was approved by the UOEH Ethics Committee in 2013 (H25-008).

\subsection{Preparation of Chemical-Albumin Complexes}

Chemical-albumin complexes were prepared as described previously with slight modifications [8]. Briefly, TDI, FA, and PA (Wako Pure Chemical Industries) were mixed with HSA (final concentration of HSA: $1 \mathrm{mM}$ ) with four ratios of the HSA to chemical concentration (i.e., 1:0, 1:1, 1:10, and 1:100) at $\mathrm{pH}$ 6.0, 8.0, and 10.8. Samples were then incubated at $37^{\circ} \mathrm{C}$ for $1 \mathrm{~h}$. The reactants were centrifuged, and the supernatants containing the chemical-HSA adducts served as diagnostic antigens.

\subsection{Detection of Chemical-Specific IgG}

Dot blots were performed as previously reported [9]. The antigens were blotted to nitrocellulose membranes (Amersham, GE Healthcare), which were then blocked with blocking buffer (Nacalai Tesque). Membranes were also blotted with human IgG (Zymed Laboratories) as positive control. Next, the membranes were incubated with serum (1:200 dilution) at room temperature for $1 \mathrm{~h}$ and then with horseradish peroxidase (HRP)-conjugated goat anti-human IgG (1:4000 dilution; Millipore) at room temperature for $1 \mathrm{~h}$. Luminescence derived from HRP was detected by ECL detection reagents (Amersham, GE Healthcare) using LuminoGraph (ATTO: WSE-6100H). The signal intensity was quantified with CS Analyzer ver 3.0 (ATTO Densitograph Software Library).

\section{Results and Discussion}

First, we examined the reactivity of HSA with TDI, PA, and FA. The primary target of TDI, PA, and FA is the lysine residue of HSA because isocyanates and carbonyl com- 
pounds have electron poor carbon and are therefore highly reactive with nucleophiles. Therefore, protein lysine residues within native HSA or HSA reacted with chemicals were quantified using TNBS. Under acidic or weakly alkaline conditions, lysine residues of HSA did not react with TDI, PA, or FA because no loss was not detected on lysine residues of HSA (Figure 1). When TDI, PA, and FA were reacted with HSA under basic conditions, lysine residues of HSA were lost in a chemical concentration-dependent manner (Figure 1), indicating that alkaline conditions promoted the reaction of HSA lysine residues with TDI, PA, and FA.

Next, we investigated structural changes in HSA-chemical adducts by native PAGE. Compared with native HSA, PA- and FA-treated HSA proteins showed major changes in electrophoretic mobility (Figure 2). In contrast, TDI only caused slight changes in the electrophoretic mobility of HSA (Figure 2). These results indicated that chemical-HSA adducts underwent structural changes triggered by loss of lysine residues. Under alkaline conditions, most lysine residues are present in the nonionized form. Therefore, diagnostic antigens can be effectively prepared at alkaline $\mathrm{pH}$ when using isocyanates and carbonyls as target chemicals.

We have previously reported that the antibody for Product-X, a mixture of epoxy resin compounds, in human serum can be detected by dot blot assay using X-HSA adducts as the antigen. Moreover, we also showed that the levels of Product-X-specific IgG in human serum are strongly correlated with the onset of dermatitis [9]. In this study, we attempted to simultaneously detect several specific antibodies against chemicals, such as TDI, PA, and FA in human serum from four workers handling plastic resin who had a history of allergy and from five unexposed workers. The participants were six men and three women with a median age (95\% confidence interval [CI]) of 42 (28 - 58) years. Serum from unexposed workers showed little or no signal on membranes blotted with chemical-HSA adducts (Figure 3). When serum from exposed workers was added to the membrane, the signal was detected at the spot blotted with chemicals that had been reacted with HSA under alkaline condition, with higher signals observed as the molar ratio of chemicals increased (Figure 3). The signal intensities for all chemical substances that had been reacted with HSA at an HSA to chemical concentration of 1:100 at $\mathrm{pH} 10.8$ were significantly higher in sera from exposed workers than in that from unexposed workers (TDI: $P=0.014$, PA: $P=0.028$, FA: $P=0.007$; Figure 4). Therefore, these data supported that chemical-specific IgG in human serum could be detected by dot blot assays and that chemical-HSA adducts generated under alkaline conditions could be used as antigens.

When using this method for epidemiological studies, it will be necessary to consider establishment of the optimal cut-off point and evaluation of signals derived from HSA alone. At least 50 samples from normal unexposed participants should be used to set the cut-off point in order to determine whether a participant has antibodies targeting certain chemicals [6]. HSA showed nonspecific signals in our detection process (data not shown). Thus, subtracting the signal of HSA alone from the conjugate signal before quantification of the data could improve performance [10]. 


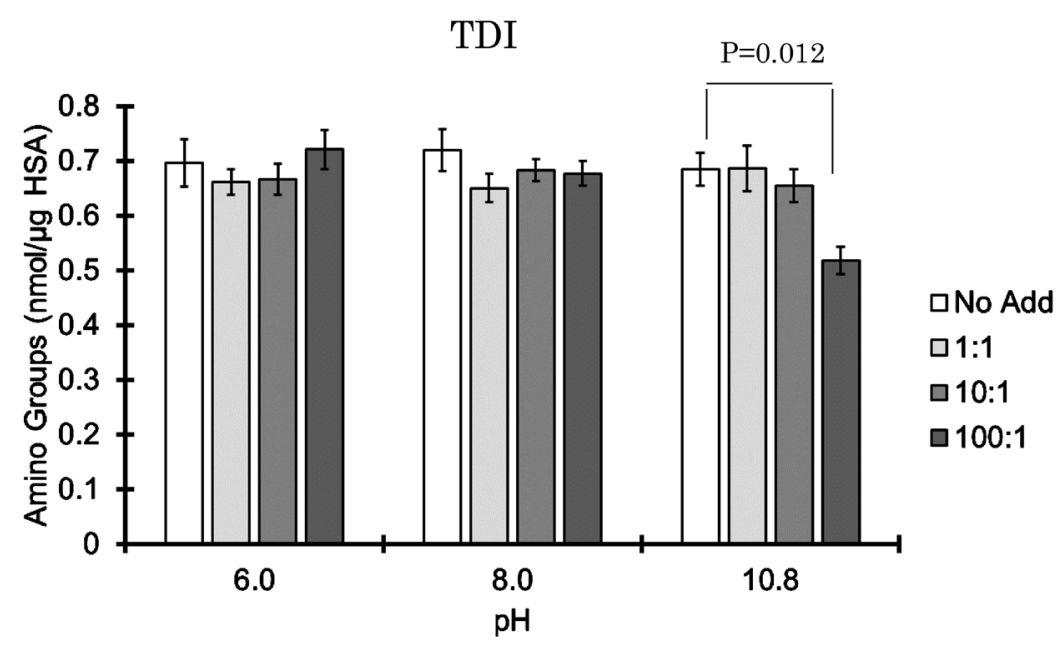

PA

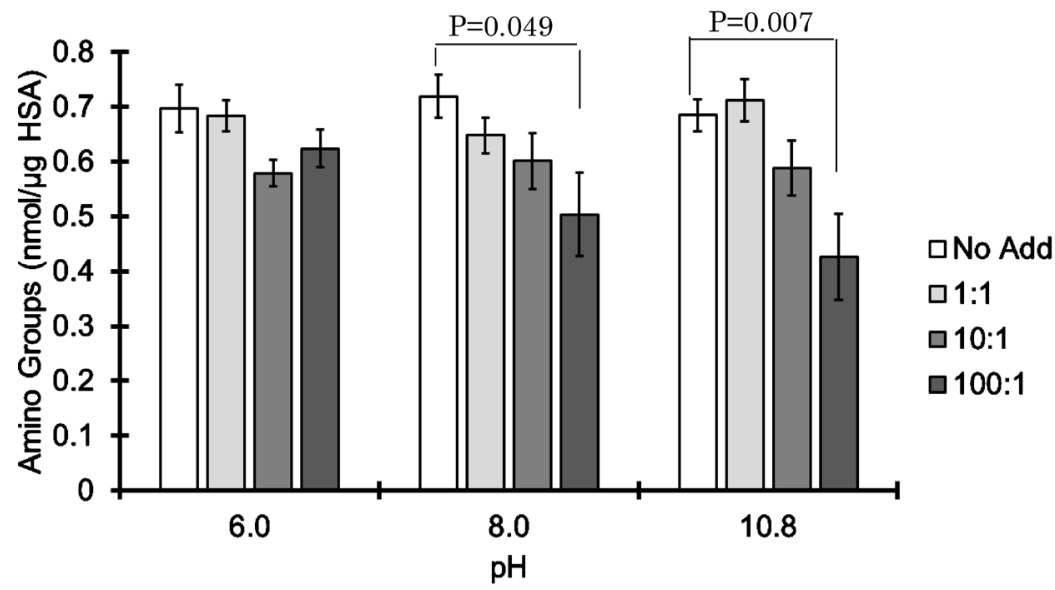

FA

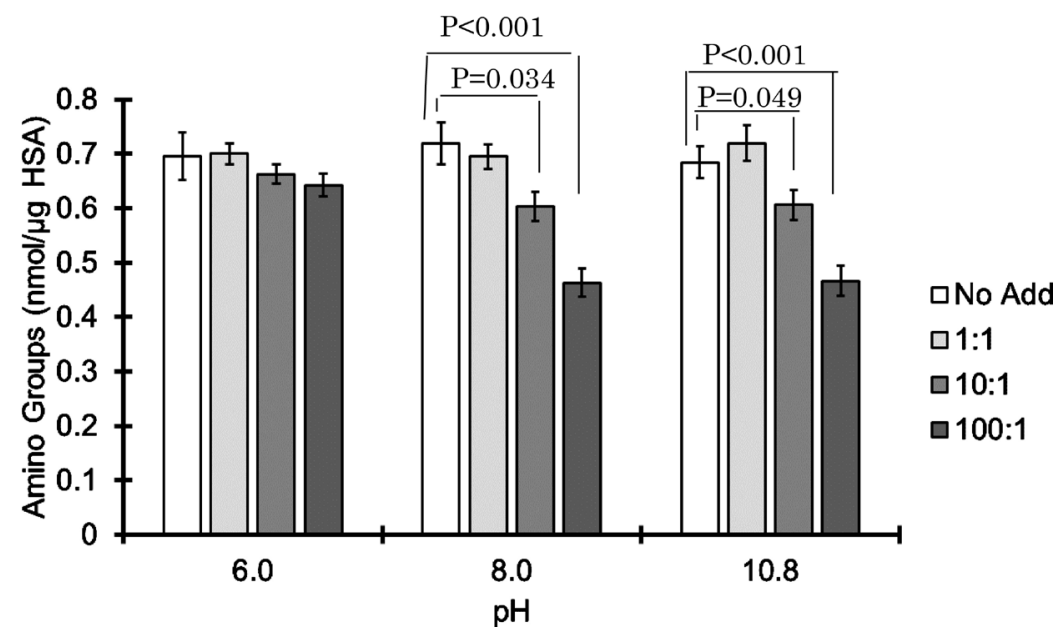

Decreases in amino groups of HSA by reaction with TDI, PA, and FA at alkaline pH. HSA was reacted with TDI, PA, and FA to form chemical-HSA adducts. The contents of primary amino groups of HSA were determined using TNBS. The values are the means \pm SEM of 10 separate experiments. $P$ values were obtained using Mann-Whitney tests.

Figure 1. Decreases in amino groups of HSA by reaction with TDI, PA and FA at alkaline pH. 


\section{Native PAGE}

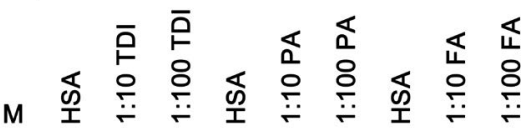

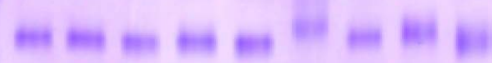

$-\infty-\infty$

Detection of structural changes in chemical-HSA adducts. HSA was reacted with TDI, PA, and FA at pH 10.6 to form chemical-HSA adducts and was then applied to native PAGE. Results are representative of three independent experiments.

Figure 2. Detection of structural changes in chemical-HSA adducts.
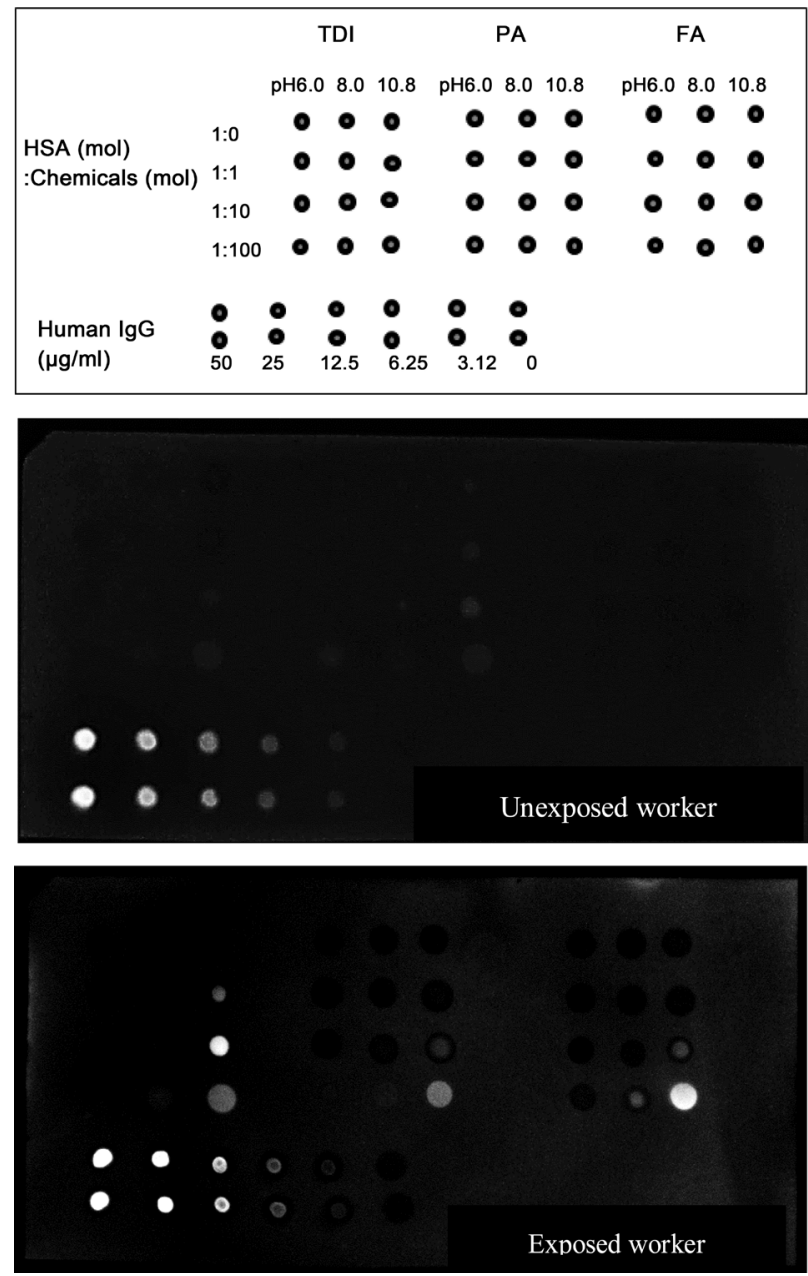

Results of dot blot assays. Samples in buffers at $\mathrm{pH}$ 6.0, 8.0, and 10.8 were blotted on membrane in the order from low mixture ratio (top) to high mixture ratio (bottom). HSA: TDI, PA, and FA at ratios of 1:0, 1:1, 1:10, and 1:100

Figure 3. Results of dot blot assay. 

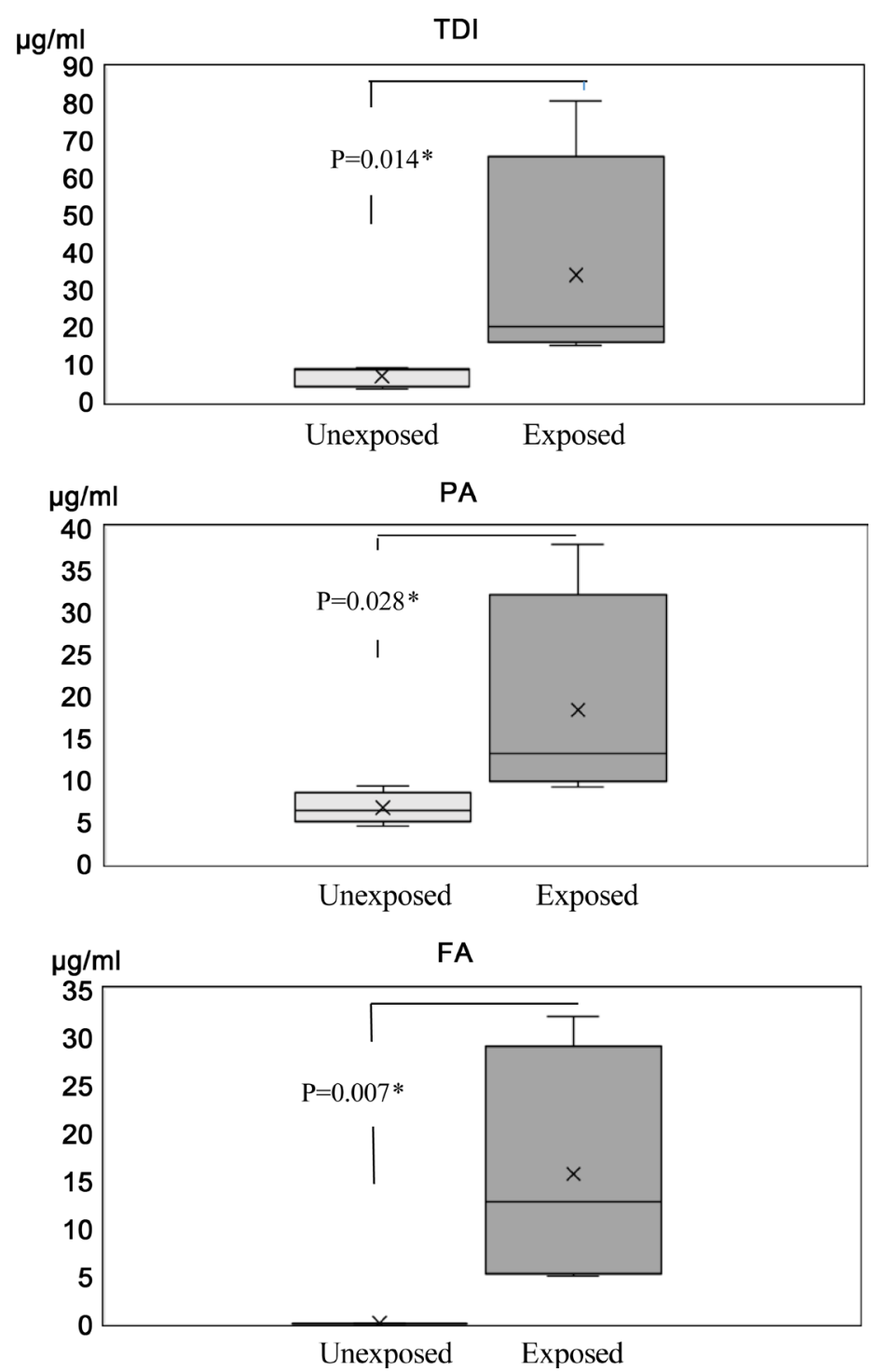

Comparison of chemical-specific IgG data between unexposed and exposed workers. $P$ values were obtained by Mann-Whitney tests. The ratio of the HSA to chemical concentration was 1:100 at $\mathrm{pH} 10.8$.

Figure 4. Comparison of chemical-specific IgG data between unexposed and exposed workers.

TDI-specific IgG and PA-specific IgG in serum have been previously detected by ELISA [11] [12]. Both ELISA and dot blotting can be used to measure chemical-specific antibodies in serum. However, in the ELISA system, it is difficult to confirm whether all chemical-HSA adducts bind to the wells of ELISA plates. In addition, although ELISA can be used to process many samples at the same time, this method is time consuming when numerous chemicals are tested. In contrast, dot blotting is advantageous because it can be used for simultaneous detection of multiple antibodies against chemicals. Moreover, because plants handling plastic resins typically use more than one type of chemical substance, exposing workers to various combinations of chemicals, simple methods are needed for analysis of multiple chemical-specific IgGs in serum. Thus, dot blotting is more suitable than ELISA in this regard. 
Isocyanate and carbonyl groups can react with protein lysine residues under alkaline conditions, leading to structural changes in HSA. A high signal was detected when workers' serum was applied to the spot with chemical-HSA adducts, which were generated under alkaline $\mathrm{pH}$ and with a high molar ratio. Namely, chemical-modified HSA, exhibiting variations in structure, had high reactivity to workers' serum. Therefore, chemical-specific IgG was clearly detected by our dot blotting method.

The workers used TDI, PA, and FA in the workplace. However, both exposed and unexposed workers may have come in contact with these chemical substances in daily life, e.g., from smoking or during hobbies, etc. In such cases, the extent and frequency of exposure would be expected to be lower than that in the workplace. However, it is necessary to consider individual exposure levels when diagnosing occupational diseases.

In this study, we succeeded in the detection of chemical-specific IgG in human serum using conjugates of carbonyl and isocyanate compounds with HSA. Isocyanate and carbonyl compounds can react with lysine residues in HSA, leading to structural changes HSA, which can cause antigenicity. Moreover, our method could apply to the detection of chemical-specific antibodies other than TDI, PA, and FA if HSA can react with other chemicals. For example, methyl methacrylate and methyl acrylate, which are raw materials used in the production of acrylic acid resin and have sensitizing potential in humans, react with cysteine residues in proteins [13]. Therefore, our dot blotting method could be expanded for simultaneous detection of additional chemical-specific antibodies in the future.

\section{Conclusion}

TDI, PA, and FA induced structural changes in HSA by reacting with lysine residues of HSA under alkaline conditions. The dot blot assay developed in this study used chemical-HSA adducts as antigens and could simultaneously detect multiple chemical-specific antibodies in human serum with high sensitivity. Our simple method is expected to contribute to the diagnosis of allergies in workers and the prevention of health hazards by harmful chemicals through determination of various chemical-specific antibodies in human serum.

\section{Acknowledgements}

This study was funded by an Industrial Disease Clinical Research Grant (to M.T.), UOEH Research Grant for Promotion of Occupational Health (to M.T.), and the JSPS KAKENHI (grant numbers 22790546 and 25860472 to M.T.).

\section{References}

[1] Plastics Europe (2011) Plastics-The Facts 2011. An Analysis of European Plastics Production, Demand and Recovery for 2010.

[2] Lithner, D., Larsson, A. and Dave, G. (2011) Environmental and Health Hazard Ranking and Assessment of Plastic Polymers Based on Chemical Composition. The Science of the Total Environment, 409, 3309-3324. https://doi.org/10.1016/j.scitotenv.2011.04.038 
[3] Palikhe, N.S., Kim, J.H. and Park, H.S. (2011) Biomarkers Predicting Isocyanate-Induced Asthma. Allergy, Asthma \& Immunology Research, 3, 21-26.

https://doi.org/10.4168/aair.2011.3.1.21

[4] de Groot, A.C., White, I.R., Flyvholm, M.A., Lensen, G. and Coenraads, P.J. (2010) Formaldehyde-Releasers in Cosmetics: Relationship to Formaldehyde Contact Allergy. Part 1. Characterization, Frequency and Relevance of Sensitization, and Frequency of Use in Cosmetics. Contact Dermatitis, 62, 2-17. https://doi.org/10.1111/j.1600-0536.2009.01615.x

[5] Kadivar, S. and Belsito, D.V. (2015) Occupational Dermatitis in Health Care Workers Evaluated for Suspected Allergic Contact Dermatitis. Dermatitis: Contact, Atopic, Occupational. Drug, 26, 177-183.

[6] Bernstein, D.I., Ott, M.G., Woolhiser, M., Lummus, Z. and Graham, C. (2006) Evaluation of antibody Binding to Diisocyanate Protein Conjugates in a General Population. Annals of Allergy, Asthma \& Immunology: Official Publication of the American College of Allergy, Asthma, \& Immunology, 97, 357-364. https://doi.org/10.1016/S1081-1206(10)60801-0

[7] Sashidhar, R.B., Capoor, A.K. and Ramana, D. (1994) Quantitation of Epsilon-Amino Group Using Amino Acids as Reference Standards by Trinitrobenzene Sulfonic Acid. A Simple Spectrophotometric Method for the Estimation of Hapten to Carrier Protein Ratio. Journal of Immunological Methods, 167, 121-127. https://doi.org/10.1016/0022-1759(94)90081-7

[8] Pham, T.T., Oyama, T., Isse, T. and Kawamoto, T. (2009) Application of Tryptophan Fluorescence to Assess Sensitizing Potentials of Chemicals. Archives of Environmental Contamination and Toxicology, 57, 427-436. https://doi.org/10.1007/s00244-009-9297-8

[9] Kawamoto, T., Tsuji, M. and Isse, T. (2015) Comparison of IgG against Plastic Resin in Workers with and without Chemical Dermatitis. BMC Public Health, 15, 930. https://doi.org/10.1186/s12889-015-2302-4

[10] Ott, M.G., Jolly, A.T., Burkert, A.L. and Brown, W.E. (2007) Issues in Diisocyanate Antibody Testing. Critical Reviews in Toxicology, 37, 567-585. https://doi.org/10.1080/10408440701419553

[11] Ye, Y.M., Kim, C.W., Kim, H.R., Kim, H.M., Suh, C.H., Nahm, D.H., et al. (2006) Biophysical Determinants of Toluene Diisocyanate Antigenicity Associated with Exposure and Asthma. The Journal of Allergy and Clinical Immunology, 118, 885-891. https://doi.org/10.1016/j.jaci.2006.06.026

[12] Rosqvist, S., Nielsen, J., Welinder, H., Rylander, L., Lindh, C.H. and Jonsson, B.A. (2003) Exposure-Response Relationships for Hexahydrophthalic and Methylhexahydrophthalic Anhydrides with Total Plasma Protein Adducts as Biomarkers. Scandinavian Journal of Work, Environment \& Health, 29, 297-303. https://doi.org/10.5271/sjweh.734

[13] Yarbrough, J.W. and Schultz, T.W. (2007) Abiotic Sulfhydryl Reactivity: A Predictor of Aquatic Toxicity for Carbonyl-Containing Alpha, Beta-Unsaturated Compounds. Chemical Research in Toxicology, 20, 558-562. https://doi.org/10.1021/tx600344a 
Submit or recommend next manuscript to SCIRP and we will provide best service for you:

Accepting pre-submission inquiries through Email, Facebook, LinkedIn, Twitter, etc.

A wide selection of journals (inclusive of 9 subjects, more than 200 journals)

Providing 24-hour high-quality service

User-friendly online submission system

Fair and swift peer-review system

Efficient typesetting and proofreading procedure

Display of the result of downloads and visits, as well as the number of cited articles

Maximum dissemination of your research work

Submit your manuscript at: http://papersubmission.scirp.org/

Or contact health@scirp.org 